\title{
La radioactivité deux-protons Un nouveau mode de désintégration des noyaux
}

Bertram Blank (blank@cenbg.in2p3.fr) et Grégory Canchel

Centre d’Études Nucléaires de Bordeaux-Gradignan, Chemin du Solarium, 33175 Gradignan Cedex

Le présent article décrit

la découverte d'une

nouvelle radioactivité,

dite " deux-protons »,

prédite théoriquement

et récemment mise en

évidence par l'étude de la

décroissance de deux noyaux

exotiques riches en protons,

le fer-45 et le zinc-54.

La découverte « indirecte »

de la radioactivité « deux-

protons » a été suivie

par la visualisation directe

des deux protons émis dans

des chambres à projection

temporelle. Ces nouvelles

données permettent une

confrontation des résultats

expérimentaux avec

les prédictions théoriques.

Ainsi, cette radioactivité

« deux-protons » commence

à se révéler un outil puissant

en recherche sur la structure nucléaire.
Comment étudier la structure du noyau atomique ? Les physiciens utilisent principalement deux outils : les collisions entre deux noyaux (cf. encadré, p. 8) et la décroissance des noyaux radioactifs. Dans cette dernière catégorie, une nouvelle méthode basée sur la radioactivité " deux-protons », devient accessible aux expériences de physique nucléaire. La décroissance des noyaux radioactifs permet d'accéder à certaines propriétés des noyaux : le temps caractéristique au bout duquel une population de noyaux est réduite de moitié (la durée de vie) et les pourcentages des différentes voies de décroissance empruntées par le noyau (les rapports d'embranchement). Bien que ces caractéristiques pour un noyau donné ne puissent pas être modifiées par l'expérimentateur, elles contiennent néanmoins des informations très riches quant à la structure nucléaire, par exemple l'arrangement des nucléons (protons et neutrons) à l'intérieur du noyau atomique ou encore leurs interactions mutuelles.

\section{Les radioactivités classiques [1]}

La radioactivité fut découverte par Henri Becquerel en 1896. Très rapidement, Pierre et Marie Curie, ainsi qu'Ernest Rutherford, réussirent à caractériser les différents types de radioactivités naturelles. L'émission par un noyau lourd, tel l'uranium ou le thorium, d'un petit noyau d'hélium-4, constitué de deux protons et de deux neutrons, fut appelée radioactivité " alpha ». La transformation, à l'intérieur du noyau, d'un neutron excédentaire en un proton, accompagnée de l'émission de deux particules légères, fut nommée radioactivité " bêta ». Plus tard, on distinguera entre la radioactivité « bêta - " que nous venons de décrire et la radioactivité «bêta + " qui transforme un proton excédentaire en un neutron. La troisième des radioactivités " classiques " est la radioactivité " gamma ". Celle-ci ne modifie pas la composition du noyau atomique, mais lui permet de perdre son tropplein d'énergie en le ramenant d'un état excité à un état de moindre énergie, qui peut être un autre état excité ou bien son état fondamental. Comme ces radioactivités se distinguent par la charge et la masse des particules émises, on peut les caractériser à l'aide de la déviation de ces particules dans un champ magnétique perpendiculaire à leur trajectoire : si la particule alpha dévie à droite, en revanche l'électron qui est associé à la radioactivité « bêta - " part à gauche et le gamma continue tout droit.

À la suite de la découverte de ces radioactivités naturelles, Irène et Frédéric Joliot-Curie créèrent la première radioactivité artificielle [2] en bombardant une feuille d'aluminium avec des particules "alpha ». Ils réussirent à produire ainsi un isotope radioactif, donc instable : le phosphore-30. Ces travaux, effectués dans les années 1930, constituent en quelque sorte, le début de l'étude des "noyaux exotiques ", noyaux comportant des rapports protons/neutrons différents de ceux trouvés dans la nature. La dernière des radioactivités considérées comme " classiques ", fut découverte par Otto Hahn et Fritz Strassmann en 1938. C'est la fission spontanée d'un noyau lourd en deux morceaux plus petits et de tailles à peu près équivalentes.

Ces radioactivités ont aujourd'hui une multitude d'applications en recherche et dans l'industrie, qui vont de la médecine jusqu'à la production d'énergie. Cependant, dès les années 1960, la théorie de la structure nucléaire avait prédit l'existence d'autres types de radioactivité. Ainsi, pour des noyaux possédant un très fort excédent de protons, le théoricien russe V. Goldanskii avait proposé deux nouveaux modes de décroissance : les radioactivités « un-proton " et « deux-protons » [3].

\section{Les radioactivités exotiques " un-proton » et " deux-protons "}

Pour les noyaux très " riches en protons ", la répulsion coulombienne agit comme un facteur déstabilisant, et les forces nucléaires qui règnent à l'intérieur du noyau atomique ne sont plus capables de lier tous les protons. Le noyau peut cependant réduire cette très grande instabilité en se débarrassant de quelques protons en trop. Il était donc prédit que les noyaux ayant un nombre impair de protons n'émettraient qu'un seul proton, tandis que pour des noyaux avec un nombre pair de protons, du fait de la composante de l'interaction nucléaire appelée "force d'appariement " qui favorise fortement l'association des nucléons par paires, l'émission d'un 


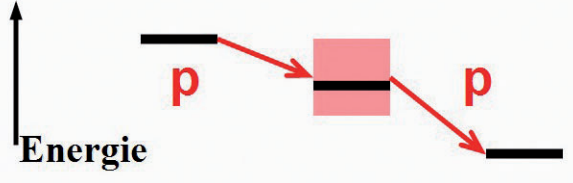

1. L'émission de deux protons peut avoir lieu de manière séquentielle (a) où un état nucléaire intermédiaire est utilisé, ou de manière simultanée (b) quand l'état intermédiaire n'est pas accessible énergétiquement. C'est ce dernier type de décroissance qui est appelé radioactivité deux-protons.

seul proton est énergétiquement interdite ; la seule possibilité qui satisfait à la loi de conservation de l'énergie est d'émettre deux protons simultanément (fig. 1b). Ainsi, l'état nucléaire obtenu après l'émission d'un seul proton a plus d'énergie que l'état de départ, alors que l'état obtenu après l'émission de deux protons en a moins.

Au début des années 1980, la radioactivité " un-proton » fut découverte au laboratoire "Gesellschaft für Schwerionenforschung " (GSI) à Darmstadt, Allemagne. Les noyaux de lutécium-151, émetteurs proton, furent produits par des réactions de fusion d'un projectile de nickel-58 avec un noyau cible de ruthénium-96, suivies de l'évaporation d'un proton et de deux neutrons (réaction de fusion-évaporation, voir encadré p. 8, fig. b). Le lutécium-151 est alors arrêté dans un détecteur semi-conducteur silicium, décroît en émettant un proton avec une énergie cinétique de 1,23 MeV et produit ainsi un noyau d'ytterbium-150.

Aujourd'hui, près de 30 noyaux émetteurs " un-proton » sont connus. La recherche concernant ces noyaux a permis de mieux comprendre la structure des noyaux exotiques très riches en protons et, entre autres, d'établir la séquence de leurs orbitales et de leurs couches nucléaires [4].

Par contre, en ce qui concerne la radioactivité " deux-protons » recherchée dès la suggestion théorique de son existence, les premières tentatives ont connu des débuts infructueux. La difficulté provient du fait que les noyaux candidats à cette radioactivité sont nettement plus difficiles à produire que les émetteurs "un-proton ». En effet, l'appariement mentionné plus haut confere une stabilité accrue aux noyaux possédant un nombre pair de protons, ce qui autorise un excès de protons nettement plus prononcé que pour les noyaux émetteurs «un-proton ». Donc, pour arriver aux noyaux instables par rapport à l'émission de deux protons, il faut créer des noyaux encore plus déficients en neutrons que pour les émetteurs "un-proton ». Et plus ce déséquilibre entre le nombre de protons et de neutrons s'accroit, plus les noyaux sont " exotiques ", donc rares et très difficiles à produire. Il a fallu attendre les années 1990 et un développement important des techniques expérimentales pour réussir à synthétiser les premiers candidats intéressants.

Dans les années 1990, des calculs théoriques de structure nucléaire identifiaient les noyaux de fer-45 (26 protons), de nickel-48 (28 protons) et de zinc-54 (30 protons) comme les candidats les plus prometteurs pour cette radioactivité.

\section{Découverte de la radioactivité "deux-protons"}

La radioactivité " deux-protons " fut découverte en 2002 [5] grâce à deux expériences réalisées sur l'étude de la décroissance du fer-45 et menées conjointement au Grand Accélérateur National d'Ions Lourds (GANIL) à Caen [6] et au GSI [7]. Les noyaux de fer-45 furent produits par des réactions dites " de fragmentation du projectile " (p. e. , dans la " cible SISSI" du GANIL - fig. 2). Dans une telle réaction, le noyau projectile, ici du nickel-58, est accéléré à une vitesse comprise entre 10\% et $50 \%$ de celle de la lumière et dirigé sur un autre noyau cible. Il existe alors une certaine probabilité pour que la cible « arrache " quelques nucléons au projectile (voir encadré p. 8, fig. d). Cette technique permet de produire des noyaux plus légers que le projectile, c'est-à-dire ayant moins de protons et/ou de neutrons, ici le fameux fer-45. Cependant, la probabilité de production varie fortement en fonction du déséquilibre entre le nombre de protons et de neutrons. Comme nous l'avons vu plus haut, les noyaux riches en protons sont difficiles à synthétiser et c'est cette raison qui rend la création de noyaux émetteurs " deux-protons " extrêmement rare. Par exemple, pour produire un seul noyau de fer-45, il faut provoquer la fragmentation de $10^{15}$ à $10^{16}$ noyaux « projectiles " stables de nickel-58.

Pour isoler les noyaux d'intérêt au milieu de l'immense majorité de noyaux produits, les expérimentateurs utilisent des séparateurs de fragments (voir, par exemple, le «spectromètre LISE3 » sur la figure 2). Ces machines tirent habilement parti de l'utilisation de champs magnétiques et électriques pour isoler les fragments recherchés. En parallèle, une parfaite identification individuelle de chaque noyau est assurée grâce à la mesure de la perte d'énergie dans les différents détecteurs et du temps que mettent les fragments à parcourir la distance de la cible aux détecteurs, le «temps de vol ». De plus, la signature de la radioactivité "deux-protons " repose sur différentes observables expérimentales : l'énergie libérée lors de la décroissance, la durée de vie du noyau émetteur, l'absence des particules légères accompagnant par exemple une désintégration bêta (voie de décroissance concurrente à la radioactivité " deux-protons ") et l'observation des caractéristiques de décroissance du noyau résultant de la radioactivité "deux-protons " (appelé " noyau fils »). Même si, prises 
séparément, ces observables ne permettent pas une conclusion unique, ensemble elles n'autorisent plus alors qu'une seule interprétation cohérente : l'existence d'un nouveau mode de désintégration de la matière nucléaire, la radioactivité "deux-protons".

La figure 3 représente le nombre de coups mesurés par intervalle de $20 \mathrm{keV}$ en fonction de l'énergie. Ce spectre montre l'existence d'un pic à une valeur de $1,14 \mathrm{MeV}$, qui coïncide justement avec l'énergie prédite par plusieurs modèles théoriques pour l'émission de deux protons par le noyau de fer-45. Notre système de détection nous a aussi permis de nous assurer qu'aucune particule légère n'était présente lors de la décroissance du fer-45 comme, par exemple, un positon qui pourrait être émis lors de la décroissance concurrente bêta.

De plus, les événements en dehors du pic sont en accord avec la décroissance du noyau fils, le chrome-43, consécutive à l'émission de deux protons par le fer-45. Enfin, la durée de vie du fer-45 est en accord avec les prédictions des modèles théoriques de la radioactivité "deux-protons".

Des résultats similaires ont été récemment obtenus pour le zinc-54 [7], un autre noyau prédit comme émetteur potentiel "deux-protons". Ces deux noyaux sont actuellement les seuls où une telle décroissance a pu être mise en évidence. Pour le nickel-48, autre noyau candidat, les indications expérimentales ne sont actuellement pas suffisamment claires pour conclure à une émission "deux-protons ».

\section{Étude du mécanisme d'émission de deux protons}

La radioactivité " deux-protons " constitue une réelle découverte. Néanmoins, le mécanisme de l'émission des deux protons est encore mal connu. S'agit-il d'une émission où les deux protons sont fortement corrélés en angle et en énergie ou a contrario d'une émission isotrope des deux protons? Les études menées jusqu'ici faisant appel à des détecteurs de type silicium ne permettaient pas aux protons d'échapper à ces détecteurs, car leur parcours dans le silicium est trop court (de l'ordre de $7 \mu \mathrm{m}$ ). Ainsi, seule leur énergie totale et l'absence d'autres particules telles que des positons de la radioactivité concurrente bêtat pouvaient être détectées. Pour étudier le mécanisme d'émission, des outils expérimentaux plus raffinés et à la pointe de la technologie devaient être mis en place.

Dans cette optique, des chambres à projection temporelle (TPC pour "time projection chamber”) ont été développées au CEN de Bordeaux-Gradignan (fig. 4) et à l'Université de Varsovie en Pologne. Dans ces chambres,

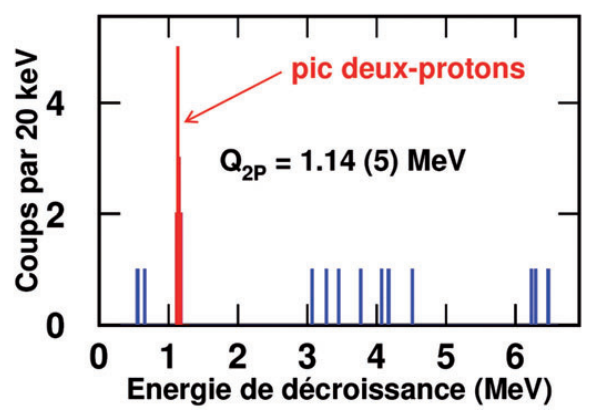

remplies d'un gaz de détection, les protons émis lors d'une radioactivité deux-protons ont un parcours suffisamment long (de l'ordre de 1,5 cm) pour permettre de les visualiser. Ils ionisent le gaz de détection, et les électrons de cette ionisation se déplacent dans le champ électrique de la chambre jusqu'à un détecteur plan à localisation. La lecture est soit électronique (version réalisée au CENBG avec une résolution de $200 \mu \mathrm{m}$ ), soit avec une caméra digitale (version polonaise). La hauteur de la trajectoire des protons (par rapport au plan de détection) est obtenue par le temps de dérive des électrons dans le gaz, qui est caractéristique de la hauteur à laquelle a lieu la création des électrons. Le start commun à toutes les voies de détection de cette mesure de temps est donné par l'arrivée des premiers électrons sur la matrice de mesure, tandis que le stop est enregistré par chaque voie individuellement, au moment de l'arrivée des électrons sur cette voie. Ainsi, seule une différence de temps est mesurée en chaque point de détection. Connaissant la vitesse de dérive (constante) des électrons dans le gaz, cette différence de temps permet de déterminer l'angle de la trajectoire des protons et ainsi cette trajectoire en trois dimensions.
3. Le pic rouge représente l'énergie correspondant à la décroissance du fer-45 par émission de deux protons; elle est mesurée à 1,14(5) MeV. Les événements à plus haute et à plus basse énergie (en bleu) sont compatibles avec la décroissance du noyau fils, le chrome-43 [6].

\section{Références}

1 - En introduction, on pourra consulter R. Bimbot, Histoire de la radioactivité, Vuibert, Paris (2006).

2 - Voir l'article de P. Radvanyi, « Frédéric Joliot-Curie et la première pile atomique française ", dans ce numéro, p. 17.

3 • V. Goldanskii, Nucl. Phys. 19 (1960) 482.

4 B. Blank, M.J.G. Borge, Prog. Part. Nucl. Phys. 60 (2008) 403.

5 - B. Blank, Physics World 21, n5 (mai 2008) 35.

6 - J. Giovinazzo et al., Phys. Rev. Lett. 89 (2002) 102501.

7 • M. Pfützner et al., Eur. Phys. J. A14 (2002) 279.

8 - B. Blank et al., Phys. Rev. Lett. 94 (2005) 232501

9 - B. Blank et al., Nucl. Instrum. Meth. B, accepté pour publication.

10 - J. Giovinazzo et al., Phys. Rev. Lett. 99 (2007) 102541

11 - K. Miernik et al., Phys. Rev. Lett. 99 (2007) 192501.

12 - L. Grigorenko et al., Phys. Rev. C64 (2001) 054002.

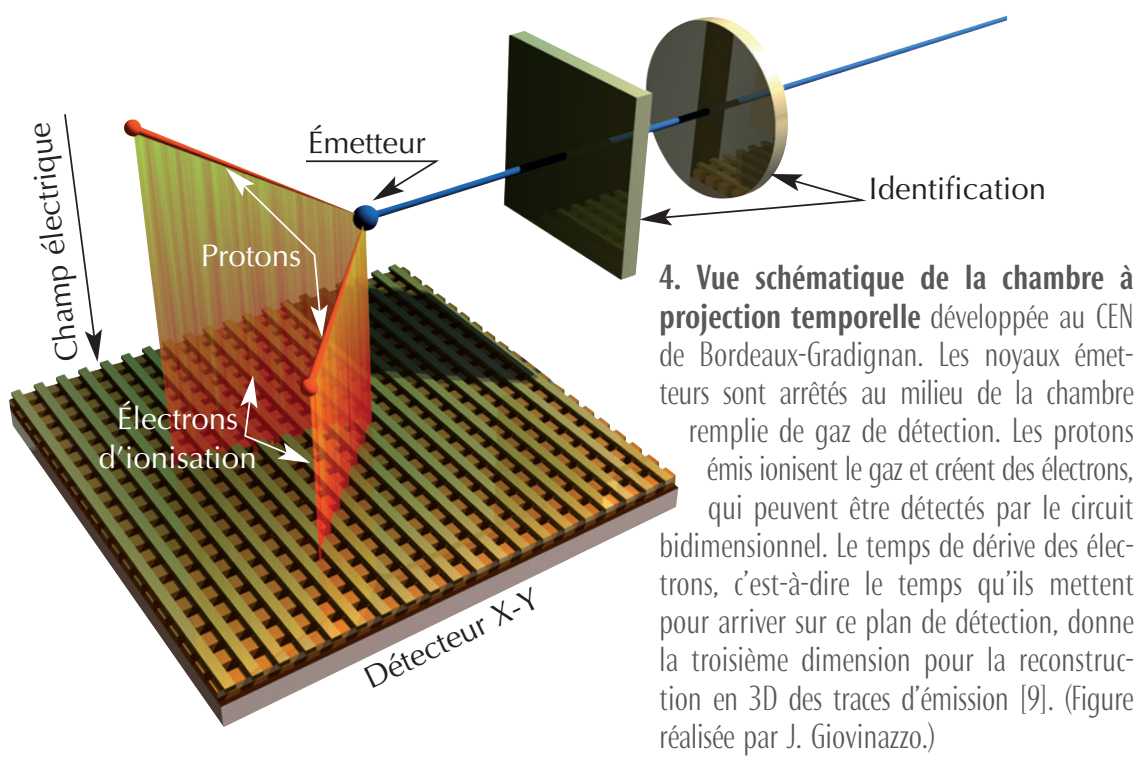



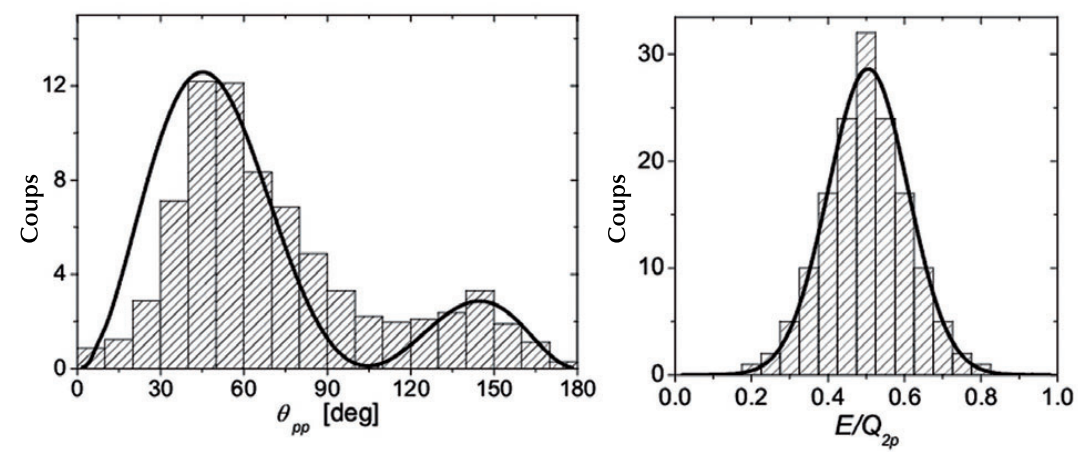

5. Distribution de l'angle entre les deux protons (à gauche) et de leur partage d'énergie totale (à droite) : ce dernier est déterminé à partir des longueurs des traces des protons [11]. L'histogramme dans chaque figure présente la mesure expérimentale, et la ligne continue est le résultat du modèle à trois corps de Grigorenko et al [12].

Ces chambres permettent donc de reconstruire la géométrie de l'émission. La longueur des traces des protons individuels, proportionnelle à leur énergie, et leur angle relatif d'émission peuvent être déterminés.

Ces mesures ont permis d'observer pour la première fois directement les deux protons émis lors de la radioactivité deux-protons du fer-45 [10]. Notamment, les résultats du groupe de Varsovie, obtenus lors d'une expérience en 2007 à Michigan State University aux USA [11], ont montré que les modèles schématiques proposés au début des études théoriques de la radioactivité deux-protons, le modèle dit « hélium-2 » qui préconise une très forte corrélation angulaire des deux protons, et le modèle de "l'espace de phase " qui ne prédit aucune corrélation et respecte uniquement la conservation de l'énergie et du moment, sont trop simples pour prendre en compte la complexité des forces nucléaires. Par contre, le modèle à trois corps développé par L. Grigorenko et al., qui modélise la désintégration avec des forces réalistes entre les deux protons et entre un proton et le noyau résiduel après l'émission des deux protons, semble être en excellent accord avec les résultats expérimentaux (fig. 5). Cette approche permet, au moins dans le cadre de ce modèle relativement simple, de déterminer des détails de la fonction d'onde des deux protons avant leur émission.

Ainsi, la nouvelle radioactivité deux-protons a ouvert une fenêtre pour sonder les forces nucléaires. En particulier, une partie de ces forces, la force d'appariement des nucléons, pourra être étudiée dans l'avenir avec ce nouvel outil.

\section{Perspectives des études avec la radioactivité deux-protons}

Pour arriver à une comparaison approfondie des résultats expérimentaux avec les modèles de la théorie nucléaire et ainsi améliorer notre compréhension de la structure du noyau atomique, d'autres études doivent être menées. Il faut dans un premier temps enrichir les résultats expérimentaux en étudiant d'autres noyaux émetteurs deux-protons. Des études avec des détecteurs de type silicium qui permettent une mesure de haute précision de l'énergie de décroissance, de la durée de vie et des différentes branches de décroissance sont à entreprendre, tout comme des mesures avec les TPC, qui nous autorisent à étudier en profondeur le mécanisme de décroissance.

En parallèle, le développement théorique doit continuer pour arriver à une description cohérente de la structure nucléaire et de la dynamique de l'émission. Évidemment, ce développement n'est pas simple et de nouvelles idées doivent être proposées pour faire de la radioactivité deux-protons un outil d'étude puissant de la structure du noyau atomique.

\section{$\rightarrow$ COLLISIONS ENTRE NOYAUX ATOMIQUES}

Pour créer en laboratoire des noyaux radioactifs qui n'existent pas dans la nature, le physicien utilise des collisions entre deux noyaux stables.

L'expérimentateur a le libre choix du couple de noyaux qu'il désire faire interagir et de l'énergie avec laquelle leur rencontre doit s'effectuer. De ces deux paramètres dépendra le type de réaction qui aura lieu et les caractéristiques des interactions. Ainsi, en utilisant par exemple un noyau très lourd tel que l'uranium comme cible ou projectile, une réaction de fission peut être provoquée. En variant l'énergie du projectile incident sur la cible, des réactions élastiques ou inélastiques peuvent se produire, conduisant dans certains cas à la fusion du projectile et de la cible, ou à la spallation/fragmentation (abrasion de quelques nucléons) de l'un des deux ou des deux à la fois.
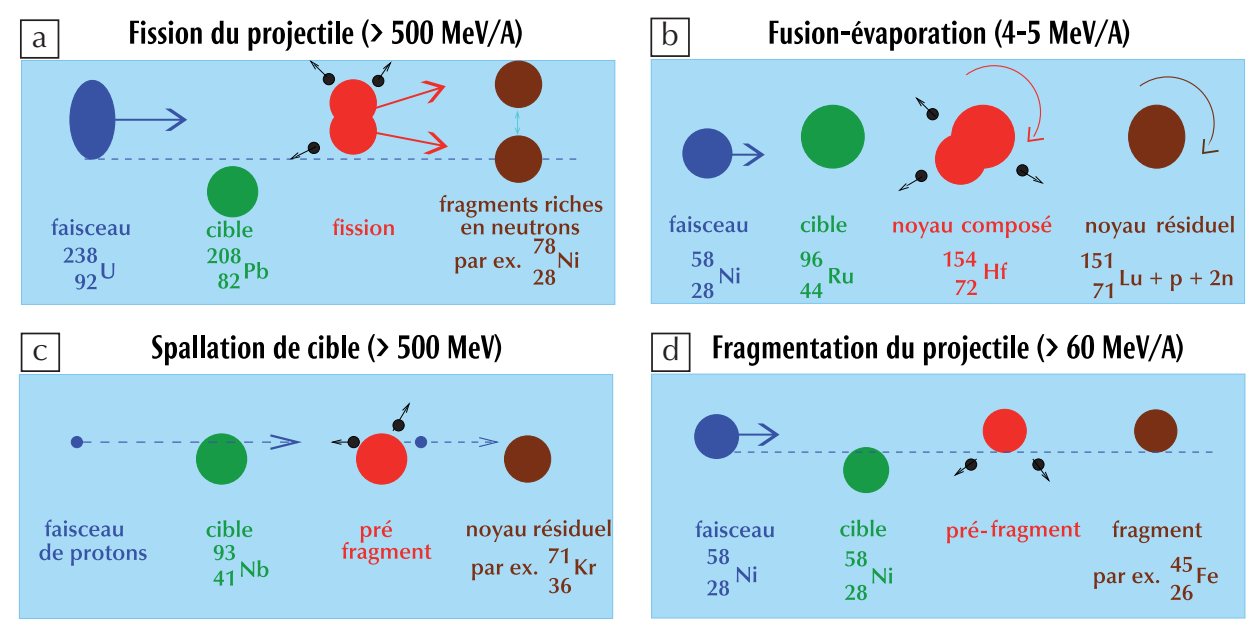

Schémas des différentes réactions nucléaires utilisées pour produire des noyaux exotiques ou pour étudier leur structure. (A : nombre de nucléons du projectile). Dans la fission (a), un noyau lourd est cassé en deux morceaux riches en neutrons, de tailles à peu près similaires. Le contraire est fait dans la fusion (b) : deux noyaux de tailles comparables s'approchent avec une vitesse juste suffisante pour vaincre la répulsion mutuelle due à la charge positive des deux noyaux. Après évaporation de particules légères (protons, neutrons ou particules alpha), le noyau final est créé. Dans la spallation (c) et la fragmentation (d), un noyau, respectivement cible ou projectile, est cassé. Avec une certaine probabilité, le noyau d'intérêt est alors produit après évaporation de quelques particules légères. 